\title{
Deep tissue, wide-field multiphoton imaging using TEMPPIX
}

\author{
Adrià Escobet-Montalbán ${ }^{1}$, Roman Spesyvtsev ${ }^{1, \dagger}$, Mingzhou Chen ${ }^{1, *}$, Wardiya Afshar \\ Saber $^{2}$, Melissa Andrews ${ }^{3}$, C. Simon Herrington ${ }^{4}$, \\ Michael Mazilu ${ }^{1}$ and Kishan Dholakia ${ }^{1}$ \\ ${ }^{1}$ SUPA, School of Physics and Astronomy, University of St. Andrews, KY16 9SS, UK. \\ ${ }^{2}$ School of Medicine, University of St. Andrews, North Haugh, St. Andrews, KY16 9FT, UK \\ ${ }^{3}$ Biological Sciences, University of Southampton, SO17 1BJ, UK \\ ${ }^{4}$ CRUK Edinburgh Centre, University of Edinburgh, Institute of Genetics and Molecular Medicine, Crewe Road \\ South, Edinburgh EH4 2XR, UK \\ ${ }^{\dagger}$ Present address: Department of Physics, SUPA and University of Strathclyde, Glasgow G4 ONG, UK \\ *E-mail: mingzhou.chen@st-andrews.ac.uk
}

\begin{abstract}
We demonstrate a new approach, temporal focusing microscopy with single-pixel detection (TEMPPIX), for wide-field multiphoton imaging through scattering media without any a priori knowledge or requirement to determine the properties of the media. (C) 2018 The Author(s)
\end{abstract}

OCIS codes: $110.0113,110.175,110.6915,180.2520$.

\section{Introduction}

TEMPPIX uses temporal focusing (TF) to project Hadamard patterns at various depths within or after a turbid layer. Such projection ensures these Hadamard patterns retain their fidelity at any given depth [1]. By collecting the emitted fluorescence in an epi-fluorescence geometry through the same turbid layer, an image of fluorescent target can be generated simply by the total intensity signal recorded by a single-pixel detector [2].

\section{Experimental setup}

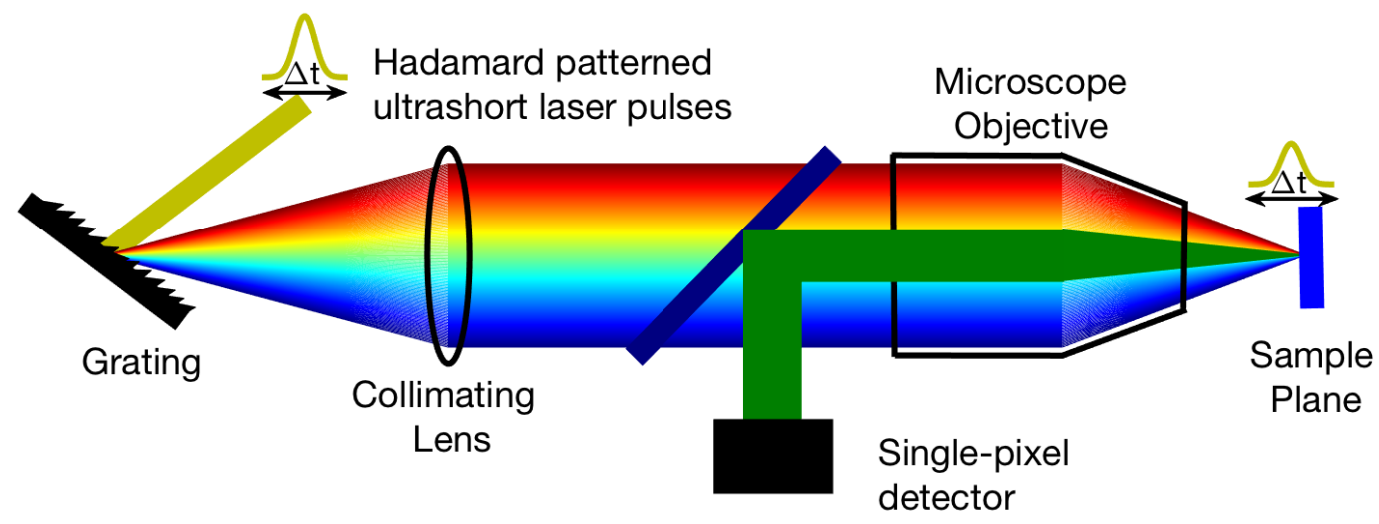

Fig. 1. The experimental schematic of TEMPPIX.

As shown in Fig. 1, the experimental TEMPPIX setup is similar to the typical TF microscopes described in the literature but adapted to perform measurements using a single-pixel detector. A femtosecond laser beam is expanded onto a spatial light modulator (not shown in the figure) that generates Hadamard patterns. The beam is then diffracted 
from a grating and the Hadamard pattens are dispersed into different spectral components and then refocused onto the sample plane after passing through the scattering medium. In this way, we temporally focused orthonormal light patterns through a turbid medium to illuminate a fluorescent microscopic sample of interest. In TEMPPIX, the size of the TF illumination plane, i.e. the field of view (FOV), is $90 \times 90 \mu \mathrm{m}^{2}$ with a maximum laser intensity being $64 \pm 5$ $\mu \mathrm{W} / \mu \mathrm{m}^{2}$. The total intensity emitted by the fluorescent sample is collected by the same objective in an epi-fluorescence geometry and detected by a single-pixel detector.

\section{Results and discussions}

Once all Hadamard patterns have been sequentially projected onto the sample plane and their total intensity measured, images are reconstructed using an orthogonal matching pursuit (OMP) algorithm [3]. One can perform both standard and compressive sensing measurements using a single-pixel detector. The performance of TEMPPIX was tested with a fluorescent target under unfixed human colon tissue or fixed rat brain tissue as scattering medium.

Fluorescent micropatterns with fine structures were generated on a thin fluorescent film and imaged through fixed rat brain slices of different thicknesses (Fig. 2). The maximum imaging depth achieved through rat brain slices was $400 \mu \mathrm{m}$ which corresponds to approximately 7 mean free paths.
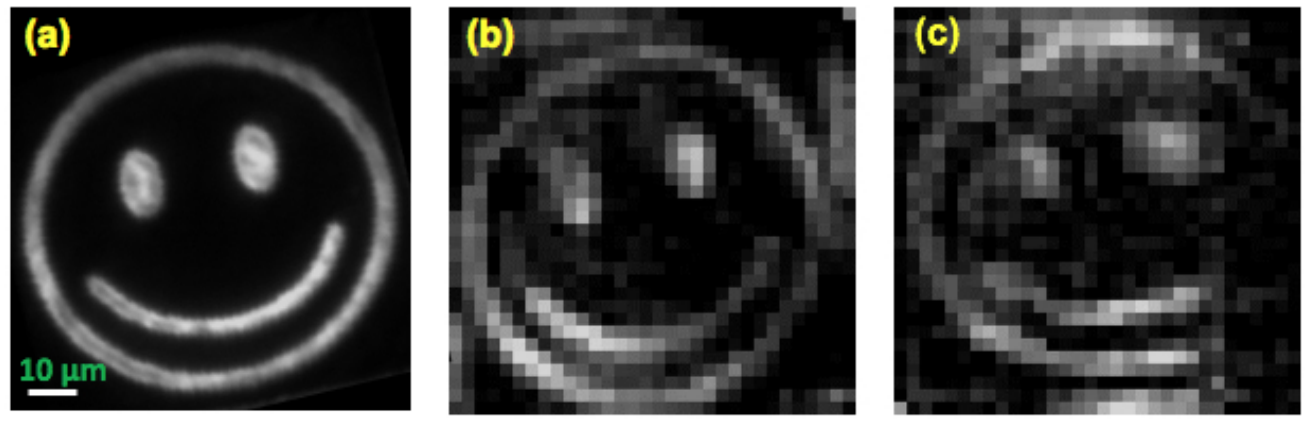

Fig. 2. (a) Reference image of the fluorescent micropattern without any scattering tissue. (b, c) Reconstructed images obtained with TEMPPIX through brain slices of $200 \mu \mathrm{m}$ and $400 \mu \mathrm{m}$, respectively. The two retrieved images were reconstructed using a full Hadamard basis containing 1024 patterns. Scale bar is $10 \mu \mathrm{m}$.

Here, we demonstrate that TEMPPIX makes it possible for the first time to obtain scan-free wide-field images of fluorescent samples through turbid tissue without any knowledge or characterization of the tissue. The epi-fluorescence geometry makes this novel approach suitable for biological applications in any standard multiphoton microscope.

\section{References}

1. E. Papagiakoumou, A. Bègue, B. Leshem, O. Schwartz, B. M. Stell, J. Bradley, D. Oron, and V. Emiliani, "Functional patterned multiphoton excitation deep inside scattering tissue," Nat. Photonics 7, 274-278 (2013).

2. V. Durán, F. Soldevila, E. Irles, P. Clemente, E. Tajahuerce, P. Andrés, and J. Lancis, "Compressive imaging in scattering media," Opt. Express 23, 14,424 (2015).

3. J. A. Tropp and A. C. Gilbert, "Signal Recovery From Random Measurements Via Orthogonal Matching Pursuit," IEEE Trans. Inf. Theory 53, 4655-4666 (2007). 